\title{
Meningococcal disease epidemiology in Australia 10 years after implementation of a national conjugate meningococcal $\mathbf{C}$ immunization programme
}

\author{
G. L. LAWRENCE ${ }^{1,2} *$ H. WANG ${ }^{2}$, M. LAHRA ${ }^{3}$, R. BOOY ${ }^{2,4}$ AND \\ P. B. MCINTYRE ${ }^{2}$ \\ ${ }^{1}$ School of Public Health and Community Medicine, University of New South Wales, Sydney, NSW, Australia \\ ${ }^{2}$ National Centre for Immunisation Research and Surveillance of Vaccine Preventable Diseases, University of \\ Sydney and The Children's Hospital at Westmead, Sydney, NSW, Australia \\ ${ }^{3}$ Australian Meningococcal Surveillance Program and WHO Neisseria Reference Laboratory, Department of \\ Microbiology, South Eastern Area Laboratory Services, The Prince of Wales Hospital, Randwick, NSW, \\ Australia \\ ${ }^{4}$ Marie Bashir Institute, University of Sydney, NSW, Australia
}

Received 15 November 2015; Final revision 11 February 2016; Accepted 21 March 2016; first published online 20 April 2016

\section{SUMMARY}

Australia implemented conjugate meningococcal $\mathrm{C}$ immunization in 2003 with a single scheduled dose at age 12 months and catch-up for individuals aged 2-19 years. Several countries have recently added one or more booster doses to their programmes to maintain disease control. Australian disease surveillance and vaccine coverage data were used to assess longer term vaccine coverage and impact on invasive serogroup $\mathrm{C}$ disease incidence and mortality, and review vaccine failures. Coverage was $93 \%$ in 1 -year-olds and $70 \%$ for catch-up cohorts. In 10 years, after adjusting for changes in diagnostic practices, population invasive serogroup $\mathrm{C}$ incidence declined $96 \%(95 \%$ confidence interval $94-98)$ to 0.4 and 0.6 cases/million in vaccinated and unvaccinated cohorts, respectively. Only three serogroup C deaths occurred in 2010-2012 vs. 68 in 2000-2002. Four $(<1 /$ million doses) confirmed vaccine failures were identified in 10 years with no increasing trend. Despite published evidence of waning antibody over time, an ongoing single dose of meningococcal $\mathrm{C}$ conjugate vaccine in the second year of life following widespread catch-up has resulted in near elimination of serogroup $\mathrm{C}$ disease in all age groups without evidence of vaccine failures in the first decade since introduction. Concurrently, serogroup B incidence declined independently by $55 \%$.

Key words: Immunization policy, immunization programme evaluation, meningococcal disease, vaccine failure.

\section{INTRODUCTION}

The global epidemiology of invasive meningococcal disease (IMD) varies considerably, both geographically

\footnotetext{
* Author for correspondence: Associate Professor G. L. Lawrence, School of Public Health and Community Medicine, University of New South Wales, Sydney, NSW 2052, Australia. (Email: g.lawrence@unsw.edu.au)
}

and temporally $[1,2]$. In recent history, serogroup B disease has predominated in Australia comprising $\sim 65 \%$ of laboratory-confirmed cases of IMD [3, 4]. However, like many other developed countries, Australia saw a rapid increase in the incidence of invasive serogroup $\mathrm{C}$ meningococcal disease around the turn of this century, although the incidence and contribution of serogroup $\mathrm{C}$ disease varied geographically, ranging from $\sim 0 \cdot 3$ cases $/ 100000$ population $(10 \%$ of 
national IMD) in Western Australia to 3.6 cases/ $100000(65 \%)$ in the southeastern state of Tasmania in $2002[3,4]$.

The development of safe and effective conjugate serogroup C vaccines in the late 1990s led to the implementation of national immunization programmes in European countries, North America and Australia using three broad strategies based on local disease epidemiology [5]. These were to (i) immunize with multiple doses in the first year of life [e.g. UK (the first country to implement a national programme), Spain, and the Canadian province of Quebec] [6-8]; (ii) give a single dose in the second year of life (e.g. Australia, The Netherlands, Canadian province of Ontario) [9-11] or (iii) immunize in adolescence (the USA, using the ACWY multivalent conjugate vaccine) [12]. Most countries that elected to routinely immunize in the first or second year of life also conducted mass catch-up immunization programmes for children aged up to 19 years to accelerate population coverage and reduce the incidence of disease in the late adolescent high-risk age group [5-11].

The Australian national meningoccocal $\mathrm{C}$ conjugate vaccine (MenCCV) immunization programme commenced in January 2003 [9] and included all three vaccines licensed at that time (i.e. NeisVacC, Meningitec, Menjugate). A single dose was introduced into the national immunization schedule at age 12 months, due to the low and stable incidence of serogroup $C$ disease in infants (only 4-6 cases/year) and the need for multiple doses in infants aged $<12$ months to achieve direct protection [13, 14]. A staged catch-up programme was conducted for the 1-19 years age group (born 1984-2001) with priority given to the 1-4 and 15-19 years age groups as they had the highest disease incidence [9]. The catch-up programme was completed for most age groups by late 2004 and extended to June 2007 to increase coverage in those aged 15-19 years who were not attending school during 2003-2004.

An important consideration for MenCCV immunization programmes in Australia and elsewhere is the long-term impact on the population incidence of invasive meningococcal $\mathrm{C}$ disease, which is related to direct and indirect protective effects dictated by the age at immunization, the likelihood of waning immunity and the extent of the initial catch-up immunization programme in terms of age groups and coverage $[5,14]$. A key consideration for all countries is whether additional booster doses are required to sustain protection and, if so, the optimal age(s) at which booster dose(s) should be given. Serological studies have found that the age at vaccination is associated with both the level and longevity of immune responses with vaccination in adolescence achieving higher titres and smaller reductions in antibody levels over time compared to younger age groups [15-18]. Countries that vaccinate in the first year of life have added a booster dose at age 12 months to the national immunization schedule due to rapidly waning immunity and a relatively high vaccine failure rate [6, 7, 19-21]. Canada, the UK and several other European countries have also now added an adolescent booster dose to their national schedules to maintain the low carriage rates achieved in catch-up campaigns in this high-risk age group $[8,21,22]$. A further consideration is the vaccine formulation to include in a booster programme, including the multivalent conjugate ACWY vaccine and recently available conjugate meningococcal $\mathrm{B}$ vaccine [23].

In this paper, we summarize and review the available data for Australian MenCCV programme evaluation indicators to 31 December 2012 and discuss the implications of these data in relation to future meningococcal immunization policy in Australia.

\section{METHODS}

\section{Vaccine coverage}

Coverage was estimated for the 1984-2011 birth cohorts for doses delivered through the funded programme. The Australian Childhood Immunisation Register (ACIR), a population-based register of $>98 \%$ of Australian children aged $<7$ years at the time of vaccination, was used to assess coverage of children born during 2002-2011, i.e. those who received the vaccine as part of the routine immunization schedule due at age 12 months [24]. Coverage in children born during 1998-2001 (aged 2-5 years in 2003) was estimated from ACIR data adjusted for underreporting using additional information obtained from a national telephone survey of parents of a random sample of 420 children born in 1999 who had no ACIR record of having received MenCCV but were recorded as up-to-date for the vaccines routinely scheduled at age 4 years. Parents were asked to read from provider-completed parent-held written records that included the date of vaccination. The detailed methods used are reported elsewhere [25].

State and territory health departments provided aggregated coverage and enrolment data for cohorts in primary school (born 1991-1997, aged 6-12 years 
in 2003) and secondary school (born 1984-1990, aged 13-19 years in 2003). Due to low secondary-school attendance, coverage for the 1984-1985 birth cohort (aged 18-19 years in 2003) was estimated separately using data provided by the Queensland, South Australia and Western Australia health departments and population denominator data estimated by the Australian Bureau of Statistics [26].

\section{Impact of the programme}

\section{Data sources}

IMD has been nationally notifiable in Australia since 1993. The National Notifiable Diseases Surveillance System (NNDSS) includes both laboratory-confirmed and clinical cases and is collated from data provided by all state and territory health departments [4, 27]. The Australian Meningococcal Surveillance Programme (AMSP) database contains data provided by the nine meningococcal reference laboratories in Australia [3]. Case definitions used by both the NNDSS and AMSP surveillance systems have changed over time. Before 1999, the AMSP contained data on culture-positive isolates only [3]. Since 2004, the NNDSS definition of a confirmed case of IMD is either isolation of Neisseria meningitidis from a normally sterile site or a clinically compatible disease plus a positive laboratory test [27]. Clinical cases are also notifiable to the NNDSS and are defined as a clinically compatible illness without laboratory evidence but with evidence of either haemorrhagic petechiae or close contact with a laboratoryconfirmed case within 30 days. Serogroup information was not available for clinical cases and for some laboratory-confirmed cases, particularly during the late 1990s and early in 2000s when non-serogroup-specific serological and nucleic acid diagnostic tests were used where culture was not possible. The proportion of cases with missing serogroup information (including clinical cases), has declined over time from $32 \%$ of cases in 2001 (6.6\% were clinical cases) to $16 \%$ in 2006 and $11 \%$ in $2012(1.9 \%$ and $0.9 \%$ were clinical cases, respectively).

\section{Disease incidence}

The impact of the MenCCV programme on the serogroup-, age- and region-specific incidence of IMD in Australia was assessed by comparing aggregated NNDSS (notification) data for the 3-year pre-programme period (2000-2002, representing the peak in notifications) and four time periods following the implementation of the programme (2003-2004, 2005-2006, 2007-2009, 2010-2012). Average annual incidence rates for the pre- and post-programme periods were used rather than single years to reduce the impact of year-to-year variation in each age group, particularly where a small number of cases was reported. Age-specific rates were estimated for the following age groups: $<1,1-4,5-14$, $15-24,25-39$ and $\geqslant 40$ years. The $2-19$ years age group included in the mass catch-up campaign in 2003 were aged 11-28 years in 2012. Region-specific rates were estimated for the southeastern states (Victoria, Tasmania - region 1), northeastern states (New South Wales, Australian Capital Territory, Queensland - region 2) and western states (South Australia, Northern Territory, Western Australia - region 3). States and territories were grouped into regions based on both geographical proximity and serogroup-specific incidence in the pre-programme period.

To account for changes in diagnostic practices over time, we assumed that cases of meningococcal disease that were missing serogroup information would have the same serogroup distribution by age group, geographical region and year of diagnosis as cases where the serogroup was recorded in the NNDSS database. Using the method described by Miller et al. [28], we adjusted the raw number of serogroup $\mathrm{C}$ and nonserogroup $\mathrm{C}$ cases accordingly and calculated the average annualized age-specific and region-specific incidence adjusted for missing serogroup information for the 2000-2002 pre-programme period and the 2010-2012 post-programme period. Incidence rate ratios (IRRs) and $95 \%$ confidence intervals (CIs) for both serogroup $\mathrm{C}$ and non-serogroup $\mathrm{C}$ meningococcal disease for the pre- and post-programme periods were calculated.

\section{Deaths}

The annual number of deaths attributed to invasive serogroup C meningococcal disease was estimated for 1999-2012. NNDSS and AMSP database records where death was recorded as the outcome and the serogroup was meningococcal $\mathrm{C}$, untyped or not recorded were linked on the variables of age, gender, state, postcode and year of diagnosis. Linked and unlinked records indicating serogroup $\mathrm{C}$ disease in one or both databases were analysed by year of death and age group. The raw (unadjusted) number of deaths is reported.

\section{Vaccine failure}

Vaccine failure was defined as the onset of laboratoryconfirmed invasive meningococcal serogroup $\mathrm{C}$ disease 
Table 1. Estimated coverage of the national MenCCV programme, Australia, January 2003 to December 2012

\begin{tabular}{llllll}
\hline \hline Programme & Birth cohort & Age in 2003 (years) & Age in 2012 (years) & Coverage (\%) & No. of doses \\
\hline Routine (at age 12 months) & $2002-2011$ & $1^{*}$ & $1-10$ & 93 & 2753600 \\
Preschool age catch-up & $1998-2001$ & $2-5$ & $11-14$ & 81 & 865600 \\
Primary school catch-up & $1991-1997$ & $6-12$ & $15-21$ & 75 & 1431950 \\
Secondary school catch-up & $1986-1990$ & $13-17$ & $22-26$ & 70 & 956400 \\
School leaver & $1984-1985$ & $18-19$ & $27-28$ & 22 & 121150 \\
Overall & $1984-2011$ & $1-19$ & $1-28$ & 76 & 6128700 \\
\hline
\end{tabular}

* Children born in 2002 were aged 1 year in 2003.

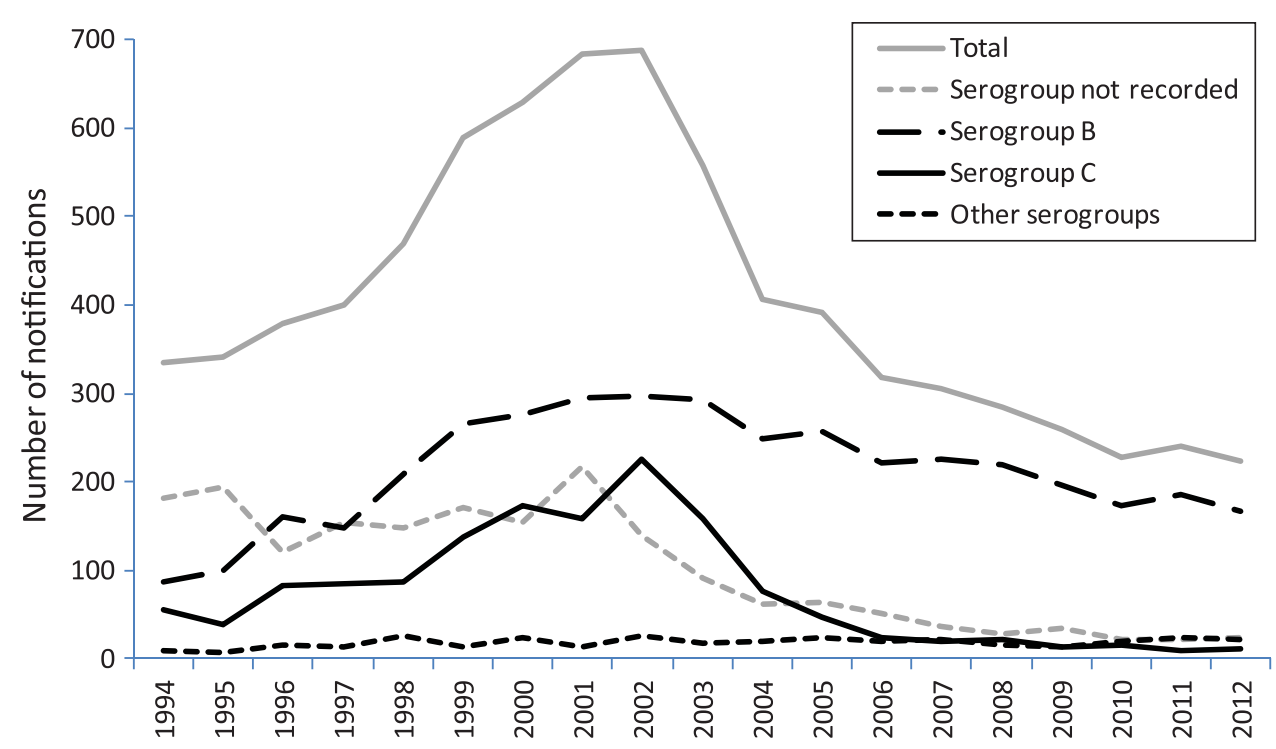

Fig. 1. Meningococcal disease notifications, by serogroup, Australia, 1994-2012. Note that 'serogroup not recorded' includes untyped isolates, serological and nucleic acid laboratory confirmatory tests as well as clinical cases.

$>14$ days after the date of vaccination. State and territory health departments were contacted to provide information about all suspected and confirmed vaccine failures recorded in the NNDSS dataset.

\section{RESULTS}

\section{Vaccine coverage}

Approximately 8.07 million people born during 1984 2011 were eligible to be immunized through the funded national MenCCV catch-up and routine immunization programmes by the end of 2012. An estimated 6.13 million $(76 \%)$ received the vaccine between 2003 and 2012, including 4.82 million eligible for the catch-up dose during 2003-2007. Coverage varied by age group and was progressively lower in older age groups (Table 1). It remained high at 93\% for the routine programme delivered at age 1 year. Across all age groups included in the catch-up programme, overall coverage was estimated to be $70 \%$ with the highest $(81 \%)$ in the pre-school age group and the lowest (22\%) in the 1984-1985 birth cohort.

\section{Epidemiology of IMD}

Annual notifications of IMD rose steeply from 1999 to peak at 687 cases (3.5/100 000 population) in 2002 (Fig. 1). The major contributors to this peak were serogroup C disease (225 cases, $1 \cdot 15 / 100000$ ) and notifications where serogroup information was not available (139 cases, $0 \cdot 71 / 100000)$. Large reductions in total meningococcal disease, serogroup $\mathrm{C}$ disease and untyped notifications were evident by the end of 2004. In 2012, there were only 11 notified serogroup $\mathrm{C}$ cases $(0 \cdot 05 / 100000$ population).

Independently, the incidence of serogroup B disease also peaked in 2002 (297 cases, $1 \cdot 5 / 100000$ population), 

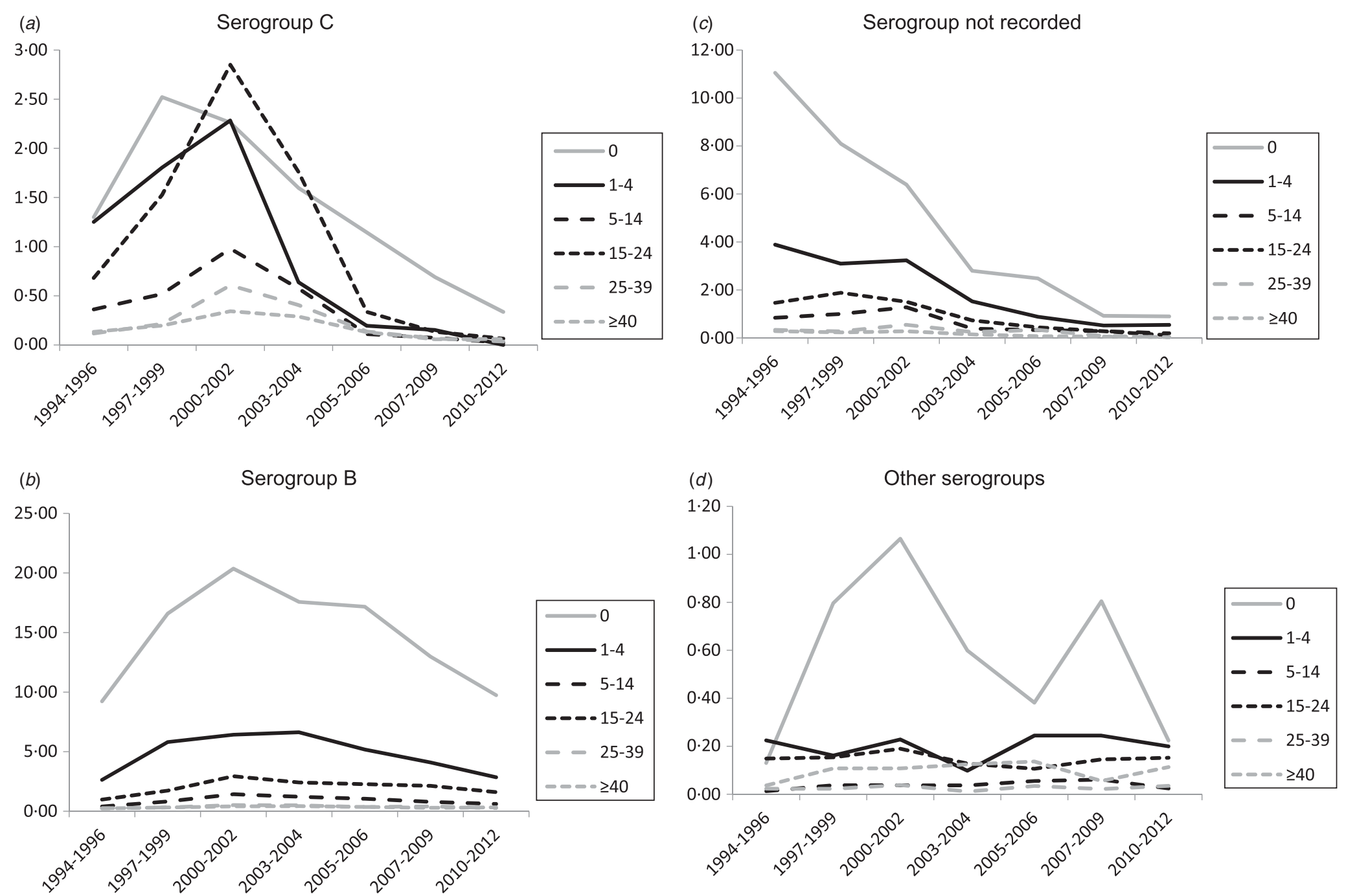

Fig. 2. Average annualized incidence of meningococcal disease/100 000 population by serogroup, time period and age group. 
Table 2. Meningoccocal disease in Australia by age group, serogroup and year, adjusted for untyped cases

\begin{tabular}{|c|c|c|c|c|c|c|c|}
\hline \multirow{3}{*}{$\begin{array}{l}\text { Age group } \\
\text { (years) }\end{array}$} & \multirow[b]{3}{*}{ Serogroup } & \multicolumn{4}{|c|}{$\begin{array}{l}\text { Adjusted* total cases and average annual incidence } \\
\text { rate/100 } 000 \text { population }\end{array}$} & \multirow{2}{*}{\multicolumn{2}{|c|}{$\begin{array}{l}\text { IRR } \\
\text { 2010-2012 vs. } \\
\text { 2000-2002 }\end{array}$}} \\
\hline & & \multicolumn{2}{|l|}{ 2000-2002 } & \multicolumn{2}{|l|}{ 2010-2012 } & & \\
\hline & & Cases (raw) & Rate & Cases (raw) & Rate & IRR & $95 \% \mathrm{CI}$ \\
\hline \multirow[t]{2}{*}{$<1$} & $\mathrm{C}$ & $22(17)$ & $2 \cdot 93$ & $3(3)$ & $0 \cdot 34$ & $0 \cdot 11$ & $0 \cdot 034-0 \cdot 38$ \\
\hline & Non-C & $204(161)$ & $27 \cdot 15$ & 97 (89) & $10 \cdot 96$ & $0 \cdot 40$ & $0 \cdot 31-0 \cdot 51$ \\
\hline \multirow[t]{2}{*}{$1-4$} & $\mathrm{C}$ & $95(70)$ & $3 \cdot 10$ & $0(0)$ & 0 & $0 \cdot 0$ & - \\
\hline & Non-C & 278 (204) & $9 \cdot 07$ & $126(107)$ & 3.59 & $0 \cdot 40$ & $0 \cdot 32-0 \cdot 49$ \\
\hline \multirow[t]{2}{*}{$5-14$} & $\mathrm{C}$ & $120(79)$ & $1 \cdot 49$ & $3(2)$ & $0 \cdot 04$ & 0.02 & $0 \cdot 01-0 \cdot 08$ \\
\hline & Non-C & $180(118)$ & $2 \cdot 24$ & $68(53)$ & $0 \cdot 82$ & $0 \cdot 37$ & $0 \cdot 28-0 \cdot 48$ \\
\hline \multirow[t]{2}{*}{$15-24$} & $\mathrm{C}$ & $282(225)$ & $3 \cdot 57$ & $6(6)$ & $0 \cdot 07$ & $0 \cdot 02$ & $0 \cdot 01-0 \cdot 04$ \\
\hline & Non-C & 309 (247) & $3 \cdot 91$ & $171(162)$ & $1 \cdot 86$ & $0 \cdot 47$ & $0 \cdot 39-0 \cdot 57$ \\
\hline \multirow[t]{2}{*}{$25-39$} & $\mathrm{C}$ & $116(79)$ & $0 \cdot 89$ & $5(5)$ & 0.03 & $0 \cdot 04$ & $0 \cdot 02-0 \cdot 10$ \\
\hline & Non-C & $106(72)$ & $0 \cdot 81$ & $48(44)$ & 0.33 & $0 \cdot 41$ & $0 \cdot 29-0 \cdot 58$ \\
\hline \multirow[t]{2}{*}{$\geqslant 40$} & $\mathrm{C}$ & $114(86)$ & $0 \cdot 46$ & $21(19)$ & $0 \cdot 07$ & $0 \cdot 15$ & $0 \cdot 09-0 \cdot 24$ \\
\hline & Non-C & $172(130)$ & 0.69 & $144(135)$ & $0 \cdot 47$ & 0.68 & $0 \cdot 54-0.85$ \\
\hline \multirow[t]{2}{*}{$1-24$} & C & $497(374)$ & $2 \cdot 61$ & $9(8)$ & $0 \cdot 04$ & 0.02 & $0 \cdot 01-0.032$ \\
\hline & Non-C & 767 (569) & $4 \cdot 03$ & $365(322)$ & 1.73 & $0 \cdot 43$ & $0 \cdot 38-0 \cdot 49$ \\
\hline \multirow{2}{*}{$\geqslant 25$} & $\mathrm{C}$ & $230(165)$ & $0 \cdot 60$ & $26(24)$ & $0 \cdot 06$ & $0 \cdot 10$ & $0 \cdot 06-0 \cdot 14$ \\
\hline & Non-C & 278 (202) & 0.73 & $192(179)$ & $0 \cdot 43$ & 0.58 & $0 \cdot 48-0 \cdot 70$ \\
\hline \multirow[t]{2}{*}{ All ages } & $\mathrm{C}$ & 749 (556) & $1 \cdot 30$ & $38(35)$ & $0 \cdot 06$ & $0 \cdot 04$ & $0 \cdot 02-0 \cdot 06$ \\
\hline & Non-C & 1249 (932) & $2 \cdot 16$ & $654(590)$ & $0 \cdot 97$ & $0 \cdot 45$ & $0 \cdot 41-0 \cdot 50$ \\
\hline
\end{tabular}

IRR, Incidence rate ratio; CI, confidence interval.

* Adjusted for untyped meningococcal disease cases.

then declined, less sharply than serogroup $\mathrm{C}$ disease, to 166 cases $(0 \cdot 73 / 100000$ population) in 2012. Other serogroups, predominantly $\mathrm{W}-135$ and $\mathrm{Y}$, remained stable during 2000-2007 at 0.11 cases/100000, declined to $0 \cdot 06 / 100000$ in 2009 and then rose to $0 \cdot 11$ in 2012 .

\section{Age distribution}

The incidence of IMD declined in all age groups after 2002 (Fig. 2). The largest reduction was in serogroup $\mathrm{C}$ incidence in all age groups included in the MenCCV programme, with smaller reductions in other age groups. Concurrent independent reductions in serogroup B disease also occurred in all age groups since 2002 (Fig. 2). The highest incidence of IMD across all time periods was in infants and is predominantly due to serogroup B (average annualized incidence of $9 \cdot 7$ cases/100 000 infants in 2010-2012).

\section{Adjusted age-specific incidence}

After adjusting for missing serogroup information (Table 2), the average annualized incidence of meningococcal serogroup C declined by $96 \%$ (95\% CI 94-97) from $1 \cdot 30 / 100000$ population in the pre-programme period (2000-2002) to $0 \cdot 06 / 100000$ in 2010-2012 (IRR 0.04, 95\% CI 0.03-0.06). During the same timeframe, non-serogroup $\mathrm{C}$ incidence declined by $55 \%$ (95\% CI 50-59) from $2 \cdot 16$ to $0 \cdot 97 / 100000$ population (IRR 0.45, 95\% CI 0.41-0.50).

The decrease in adjusted serogroup $\mathrm{C}$ disease incidence was significant across all age groups (Table 2) particularly in the 1-4 and 15-24 years age groups. The smallest percentage reduction was in the $<1$ year age group which was not included in the immunization programme. Comparing all age groups included in the immunization programme (i.e. 1-24 years $v s .<1$ year and $\geqslant 25$ years) indicates that the reduction in incidence was significantly larger in the vaccinated age groups based on non-overlapping $95 \%$ CIs (Table 2).

\section{Geographical distribution}

The distribution of serogroup B and serogroup C IMD has differed markedly across Australia and over time [2-4, 27]. Between 1994 and 1999, the majority of isolates for all states and territories was 
Table 3. Meningococcal disease in Australia by geographical region, serogroup and year adjusted for untyped cases

\begin{tabular}{|c|c|c|c|c|c|c|c|}
\hline \multirow[b]{3}{*}{ Geographical region } & \multirow[b]{3}{*}{ Serogroup } & \multicolumn{4}{|c|}{$\begin{array}{l}\text { Adjusted* total cases and average annual incidence } \\
\text { rate/100 } 000 \text { population }\end{array}$} & \multirow{2}{*}{\multicolumn{2}{|c|}{$\begin{array}{l}\text { IRR } \\
\text { 2010-2012 vs. } \\
\text { 2000-2002 }\end{array}$}} \\
\hline & & \multicolumn{2}{|l|}{$2000-2002$} & \multicolumn{2}{|l|}{$2010-2012$} & & \\
\hline & & Cases (raw) & Rate & Cases (raw) & Rate & IRR & $95 \% \mathrm{CI}$ \\
\hline \multirow{2}{*}{$\begin{array}{l}\text { Region } 1 \\
\text { (Vic, Tas) }\end{array}$} & $\mathrm{C}$ & $337(253)$ & $2 \cdot 15$ & $5(5)$ & $0 \cdot 03$ & $0 \cdot 01$ & $0 \cdot 005-0.03$ \\
\hline & Non-C & 265 (199) & 1.69 & $146(140)$ & $0 \cdot 80$ & $0 \cdot 48$ & $0 \cdot 39-0.58$ \\
\hline \multirow{2}{*}{$\begin{array}{l}\text { Region } 2 \\
\text { (ACT, NSW, Qld) }\end{array}$} & $\mathrm{C}$ & $347(250)$ & $1 \cdot 11$ & $24(21)$ & $0 \cdot 07$ & $0 \cdot 06$ & $0.04-0.09$ \\
\hline & Non-C & $689(495)$ & $2 \cdot 20$ & $370(315)$ & $1 \cdot 02$ & $0 \cdot 46$ & $0 \cdot 41-0 \cdot 53$ \\
\hline \multirow{2}{*}{$\begin{array}{l}\text { Region } 3 \\
\text { (NT, SA, WA) }\end{array}$} & $\mathrm{C}$ & $65(53)$ & $0 \cdot 60$ & $9(9)$ & 0.07 & $0 \cdot 12$ & $0 \cdot 06-0 \cdot 24$ \\
\hline & Non-C & $295(238)$ & $2 \cdot 72$ & $138(135)$ & $1 \cdot 09$ & $0 \cdot 40$ & $0 \cdot 33-0 \cdot 49$ \\
\hline \multirow[t]{2}{*}{ Australia } & $\mathrm{C}$ & $749(556)$ & $1 \cdot 30$ & $38(35)$ & $0 \cdot 06$ & $0 \cdot 04$ & $0 \cdot 02-0 \cdot 06$ \\
\hline & Non-C & $1249(932)$ & $2 \cdot 16$ & $654(590)$ & 0.97 & $0 \cdot 45$ & $0 \cdot 41-0 \cdot 50$ \\
\hline
\end{tabular}

Vic, Victoria; Tas, Tasmania; ACT, Australian Capital Territory; NSW, New South Wales; Qld, Queensland; NT, Northern Territory; SA, South Australia; WA, Western Australia.

* Adjusted for untyped meningococcal disease cases.

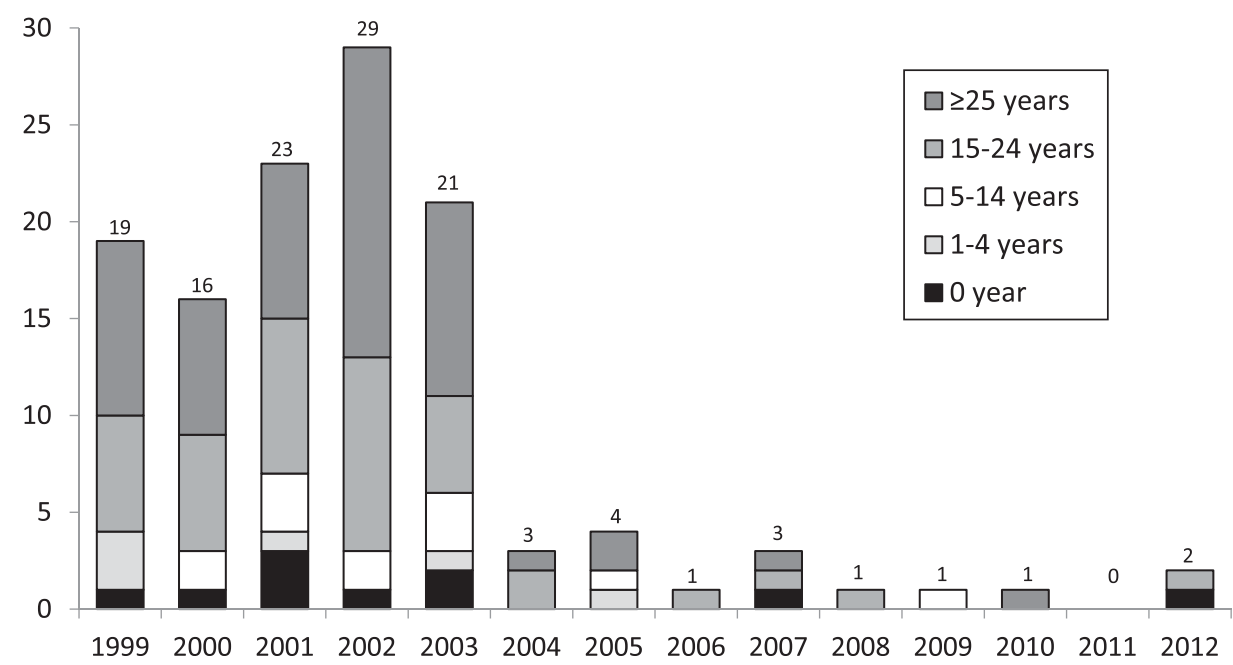

Fig. 3. Estimated number of deaths due to invasive serogroup $\mathrm{C}$ meningococcal disease by age group and year of death, Australia, 1999-2012.

serogroup B, accounting for $60-70 \%$ of the total. This changed between 2000 and 2002 when the incidence of serogroup $\mathrm{C}$ disease increased in the eastern regions of the country and particularly in region 1 (Table 3) where it became substantially higher than serogroup B incidence. In contrast, serogroup C incidence remained low and stable in the western jurisdictions (region 3) where serogroup B continued to dominate.

The adjusted incidence of both serogroup $\mathrm{C}$ and non-serogroup $\mathrm{C}$ disease declined in all geographical regions between the 2000-2002 and 2010-2012 periods, most notably in region 1 (Table 3 ).

\section{Serogroup C mortality}

For the period 1999-2012, 126 deaths attributed to meningococcal serogroup $\mathrm{C}$ were recorded in one or both of the NNDSS and AMSP databases with 61 (48\%) deaths recorded in both databases. Annual serogroup $\mathrm{C}$ deaths declined from a peak of 29 deaths in 2002 to an average of one death annually in 2008-2012 (Fig. 3). Deaths 
declined in all age groups. For the $<1$ year age group, not included in the programme, six deaths attributed to serogroup C occurred during the 4 years 19992002 compared to four deaths in the 10 -year period 2003-2012.

\section{Vaccine failures}

Between 2003 and 2012, a total of 145 serogroup C cases of IMD were reported for the birth cohorts included in the MenCCV programme. Four confirmed cases of vaccine failure were identified in immunocompetent people which amounted to $<1$ vaccine failure/ million vaccine doses administered. Of these four confirmed cases, two were vaccinated in the routine programme at ages 12 and 13 months and had disease onset 2 years and 4 years after vaccination, respectively. Two cases were vaccinated at ages 11 and 13 years in 2003; both had disease onset in 2007, 4 years after vaccination. A fifth case occurred in a child subsequently diagnosed with an immunological deficiency who was vaccinated at age 3 years and diagnosed 8 months later as part of a family disease cluster [29]. All identified cases of vaccine failure survived.

\section{DISCUSSION}

The Australian MenCCV programme achieved high (93\%) coverage for the routine childhood component at age 12 months and moderate $(70 \%)$ coverage for the catch-up programme. As seen in other countries where national MenCCV programmes have been implemented $[5-8,10,11,21]$, the impact of the programme on the incidence of invasive serogroup $\mathrm{C}$ meningococcal disease in Australia was substantial across all age groups and geographical regions. This must be interpreted in the context of changing diagnostic practices (e.g. less frequent lumbar puncture, increasing use of PCR) and a concurrent 55\% reduction in the incidence of non-serogroup $\mathrm{C}$ disease between the pre- and post-programme periods, which may reflect known periodic fluctuations in the incidence of meningococcal disease in Australia and globally, and perhaps also behaviour change in smoking $[1,2,30]$. As reported from other countries [6-8,31], there is no evidence of serogroup replacement as a result of a mass programme targeting serogroup $\mathrm{C}$ disease. Like The Netherlands, which also vaccinates after the first year of life, vaccine failures are rare compared to the rates seen in countries that have vaccinated in the first year of life [19-21, 32].
The study reported here has strengths and limitations. Limitations of the study are those inherent in the use of data collected through passive surveillance systems, particularly under-reporting of cases of invasive meningococcal disease and changes in data completeness over time (e.g. increased completeness of reporting of cases and serogroup information following implementation of the meningococcal $\mathrm{C}$ immunization programme). The latter would cause underestimation of changes in morbidity and mortality associated with serogroup $\mathrm{C}$ disease following the implementation of the programme as well that associated with non-serogroup $\mathrm{C}$ invasive meningococcal disease over the same time period.

Immunization coverage among cohorts born 1984 1998 can be considered to be minimum estimates as vaccines delivered by general practitioners outside of the main programme to those aged $>7$ years were not routinely recorded and collected by most Australian jurisdictions. Further, doses administered to all age groups prior to the nationally funded programme, particularly in geographical region 1 where pre-programme incidence was highest, could not be estimated. The lack of vaccination status being recorded for notified cases of serogroup $\mathrm{C}$ prevented estimation of vaccine effectiveness. Our assumption that the distribution of cases where serogroup information was not available was the same as for cases where it was recorded is untested but we suggest reasonable as there is no evidence that particular serogroups are more or less likely to be diagnosed.

A major strength of the study is that we were able use two data sources to estimate the number of deaths directly attributed to serogroup $\mathrm{C}$ disease. Moreover, adjustment for cases where serogroup information was missing allowed us to account for changes in diagnostic practices over time to minimize confounding. It also allowed us to compare the reduction in meningococcal serogroup $\mathrm{C}$ incidence between the pre- and post-programme periods relative to the concurrent independent secular reduction in non-serogroup $\mathrm{C}$ incidence that occurred in Australia during the postprogramme period. Prior to the implementation of the immunization programme in 2003 , the incidence of serogroup $\mathrm{C}$ disease in New South Wales (region 2) had started to decline [33] suggesting that some of the reduction in serogroup $\mathrm{C}$ incidence between the pre- and post programme periods may be related to the natural variation in serogroup incidence and/or to preprogramme vaccine uptake, i.e. that impact of the immunization programme on the reduction in serogroup $\mathrm{C}$ incidence may be overestimated to some extent. 
It should be noted that widespread herd effects are apparent in age groups not targeted by the programme. This is likely to be due to lower throat carriage rates of serogroup $\mathrm{C} N$. meningitidis at the population level [34]. The UK, Dutch and Canadian data have shown that mass immunization of the adolescent population, where carriage rates are highest, led to rapid and significant herd immunity in all unvaccinated age groups $[6,8,10,11]$, and it would be reasonable to assume that this has also occurred in Australia, i.e. a substantial vaccine effect in addition to a possible natural reduction in incidence nationally.

In terms of ongoing control of meningococcal disease, the key areas to consider are population vaccination coverage, herd immunity and throat carriage of $N$. meningitidis as well as waning immunity in those who have been vaccinated, and whether a booster dose will need to be added to the immunization programme in the future.

The available data reported here support continued use of the current schedule in Australia with a single dose at age 12 months as there is good control of MenCCV in infants aged $<1$ year. A booster dose has not been implemented at this stage, although the need for this must be monitored. A booster dose may be required prior to [16] or in early adolescence $[20,21]$ in the future before the cohorts immunized in early childhood reach late adolescence when risk of IMD rises rapidly due to increased risk behaviours [35] and higher rates of carriage of $N$. meningitidis. Preliminary analysis of Australian serosurvey data collected during 2007-2008 support previously published studies $[15,17,18]$ finding that higher antibody titres occurred in those immunized in adolescence compared to younger age groups. Mathematical modelling, using national serosurvey data, ongoing laboratory surveillance of invasive isolates, surveillance of vaccine failures and serum antibody protection will help inform decisions regarding addition of a booster dose of meningococcal $\mathrm{C}$ vaccine to the Australian national immunization schedule.

\section{CONCLUSION}

In the 10 years following the implementation of the national MenCCV immunization programme in Australia, the incidence of meningococcal $\mathrm{C}$ disease has declined substantially, by $96 \%$, alongside a natural independent reduction in meningococcal B disease of $55 \%$ during the same period. The largest reductions in serogroup $\mathrm{C}$ incidence are seen in the age groups targeted by the programme and in the geographical regions that had the highest pre-programme incidence. Like The Netherlands, which also vaccinates early in the second year of life, Australia has seen very few vaccine failures in immunocompetent children. A booster dose of MenCCV-containing vaccine has not yet been added to the immunization schedule. Surveillance continues.

\section{ACKNOWLEDGEMENTS}

The National Centre for Immunisation Research and Surveillance (NCIRS) is supported by the Australian Government Department of Health, the New South Wales Ministry of Health and the Children's Hospital at Westmead. The Australian Meningococcal Surveillance Programme (AMSP) is a product of the National Neisseria Network and is funded by the Australian Government Department of Health.

We thank Athena Limnos of the AMSP for providing data to allow estimation of deaths due to meningococcal disease; Brynley Hull (NCIRS) for providing coverage data for the routine programme and Dr Jane Jelfs for assistance in re-coding data and assisting with follow-up of vaccine failures. We are grateful to staff of state and territory health departments for providing coverage data for older age groups, and health department staff who provided confirmatory information on presumptive cases of vaccine failure.

\section{DECLARATION OF INTEREST}

None.

\section{REFERENCES}

1. Halperin SA, et al. The changing and dynamic epidemiology of meningococcal disease. Vaccine 2012; 30S: B26-36.

2. Patel MS. Australia's century of meningococcal disease: development and changing ecology of an accidental pathogen. Medical Journal of Australia 2007; 186: 136-141.

3. Australian Meningococcal Surveillance Programme. Annual report of the Australian meningococcal surveillance programme, 2002. Communicable Diseases Intelligence 2003; 27: 196-208.

4. Brotherton J, et al. Vaccine preventable diseases and vaccination coverage in Australia 2001 to 2002. Communicable Diseases Intelligence 2004; 28 (Suppl. 2): vii-S116.

5. Chiappini E, et al. Serogroup C Neisseria meningitidis invasive infection: analysis of the possible vaccination strategies for a mass campaign. Acta Paediatica 2010; 99: 1609-1614. 
6. Campbell $\mathbf{H}$, et al. Meningococcal $\mathrm{C}$ conjugate vaccine: the experience in England and Wales. Vaccine 2009; 27S: B20-B29.

7. Garrido-Estepa M, et al. Changes in meningococcal C epidemiology and vaccine effectiveness after vaccine introduction and schedule modification. Vaccine 2014; 32: 2604-2609.

8. De Wals $\mathbf{P}$, et al. Effectiveness of serogroup $\mathrm{C}$ meningococcal conjugate vaccine-a 7-year follow-up in Quebec, Canada. Pediatric Infectious Disease Journal 2011; 30: 566-569.

9. Cohen NJ. Introduction of the National Meningococcal C Vaccination Program. Communicable Diseases Intelligence 2003; 27: 161-2.

10. De Voer RM, et al. Immunity against Neisseria meningitidis serogroup $\mathrm{C}$ in the Dutch population before and after introduction of the meningococcal $\mathrm{C}$ conjugate vaccine. PLoS ONE 2010; 5: e12144.

11. Kinlin LM, et al. Rapid identification of herd effects with the introduction of serogroup $\mathrm{C}$ meningococcal conjugate vaccine in Ontario, Canada, 2000-2006. Vaccine 2009; 27: 1735-1740.

12. MacNeil JR, Cohn A, Zell E. Early estimate of the effectiveness of quadrivalent meningococcal conjugate vaccine. Pediatric Infectious Disease Journal 2011; 30: 451-455.

13. Richmond P, et al. Evaluation of de-O-acetylated meningococcal $\mathrm{C}$ polysaccharide-tetanus toxoid conjugate vaccine in infancy: reactogenicity, immunogenicity, immunologic priming, and bactericidal activity against O-acetylated and de-O-acetylated serogroup C strains. Infection and Immunity 2001; 69: 2378-2382.

14. Borrow R, et al. Effectiveness of meningococcal serogroup C vaccine programmes. Vaccine 2013; 38: 4477-4486.

15. De Voer RM, et al. Age-related immunity to meningoccoal serogroup $\mathrm{C}$ vaccination: an increase in the persistence of $\mathrm{IgG} 2$ correlates with a decrease in avidity of IgG. PLoS ONE 2011; 6: e23497.

16. Booy R, et al. Five-year antibody persistence and safety following a single dose of combined Haemophilus Influenzea type B-Neisseria meningitidis serogroup C-tetantus toxoid conjugate vaccine in Hib-primed toddlers. Pediatric Infectious Disease Journal 2015; 34: 1379-1384.

17. Snape MD, et al. Seroprotection against serogroup C meningococcal disease in adolescents in the United Kingdom: an observational study. British Medical Journal 2008; 7659; 1487-1491.

18. De Whalley PC, et al. Long-term seroprotection after an adolescent booster meningococcal serogroup $\mathrm{C}$ vaccination. Archives of Disease in Childhood 2013; 98: 686-691.

19. Trotter CL, Ramsay ME. Vaccination against meningococcal disease in Europe: review and recommendations for the use of conjugate vaccines. FEMS Microbiology Reviews 2007; 31: 101-107.

20. Khatami A, et al. Maintenance of immune response throughout childhood following serogroup $\mathrm{C}$ meningococcal conjugate vaccination in early childhood. Clinical and Vaccine Immunology 2011; 18: 2038-2042.
21. Kaaijk $\mathbf{P}$, et al. Is a single dose of meningococcal serogroup $\mathrm{C}$ conjugate vaccine sufficient for protection? The experience of the Netherlands. BMC Infectious Diseases 2012; 12: e35, 1-6.

22. Donovan H, Bedford H. Immunisation: changes in the UK for children and young people. Nursing Children and Young People 2013; 25: 16-20.

23. O'Ryan M, et al. A multi-component meningococcal serogroup B vaccine (4CMenB): the clinical development program. Drugs 2014; 74: 15-30.

24. Hull BP, et al. Immunisation coverage in Australia corrected for under-reporting to the Australian Childhood Immunisation Register. Australian and New Zealand Journal of Public Health 2003; 27: 533-538.

25. Lawrence GL, et al. Measles vaccination coverage among five-year-old children: implications for disease elimination in Australia. Australian \& New Zealand Journal of Public Health 2003; 27: 413-418.

26. Australian Bureau of Statistics. Schools Australia 2005. (Report 4221.0). Canberra; Australian Government Printing Service, 2006 (http://www.abs.gov.au/AUSS TATS/abs@.nsf/DetailsPage/4221.02005? OpenDocument). Accessed November 2015.

27. Chiu C, et al. Vaccine preventable diseases and vaccination coverage in Australia, 2005 to 2007. Communicable Diseases Intelligence 2010; 34: Si-167.

28. Miller E, et al. Herd immunity and serotype replacement 4 years after seven-valent pneumococcal conjugate vaccination in England and Wales: an observational cohort study. Lancet Infectious Diseases 2011; 11: 760-768.

29. Shaw KA, et al. Meningococcal vaccine failure in conjunction with an unusual meningococcal cluster in southern Tasmania. Communicable Diseases Intelligence 2005; 29: 159-63.

30. Rashid H, Booy R. Passive smoking, invasive meningococcal disease and preventive measures: a commentary. BMC Medicine 2012; 10: 160.

31. Trotter CL, et al. No evidence of capsule replacement following mass immunisation with meningococcal serogroup C conjugate vaccines in England and Wales. Lancet Infectious Diseases 2006; 6: 616-617.

32. Campbell $\mathbf{H}$, et al. Updated post-licensure surveillance of the meningococcal $\mathrm{C}$ conjugate vaccine in England and Wales: effectiveness, validation of serological correlates of protection, and modelling predictions of the duration of immunity. Clinical and Vaccine Immunology 2010; 17: 840-847.

33. Passmore E, Ferson MJ, Tobin S. Meningococcal disease in NSW, 1991-2011: trends in relation to meningococcal C vaccination. NSW Public Health Bulletin 2013; 24: 119-124.

34. Maiden MC, Stuart JM, UK Meningococcal Carriage Group. Carriage of serogroup $\mathrm{C}$ meningococci 1 year after meningococcal $\mathrm{C}$ conjugate polysaccharide vaccination. Lancet 2002; 359: 1829-1831.

35. Tully J, et al. Risk and protective factors for meningococcal disease in adolescents: matched cohort study. British Medical Journal 2006; 332: 445-450. 